\title{
Degenerate higher order scalar-tensor theories beyond Horndeski and disformal transformations
}

\author{
Jibril Ben Achour, 1,2 , * David Langlois, 1 , \\ ${ }^{1}$ Laboratoire APC - Astroparticule et Cosmologie, \\ Université Paris Diderot Paris 7, 75013 Paris, France \\ ${ }^{2}$ Department of Physics 83 Center for Field Theory and Particle Physics, \\ Fudan University, 200433 Shanghai, China \\ ${ }^{3}$ Laboratoire de Mathématiques et Physique Théorique, \\ Université François Rabelais, Parc de Grandmont, 37200 Tours, France
}

(Dated: February 29, 2016)

\begin{abstract}
We consider all degenerate scalar-tensor theories that depend quadratically on second order derivatives of a scalar field, which we have identified in a previous work. These theories, whose degeneracy in general ensures the absence of Ostrogradski instability, include the quartic Horndenski Lagrangian as well as its quartic extension beyond Horndeski, but also other families of Lagrangians. We study how all these theories transform under general conformal-disformal transformations and find that they can be separated into three main classes that are stable under these transformations. This leads to a complete classification modulo conformal-disformal transformations. Finally, we show that these higher order theories include mimetic gravity and some particular khronometric theories. They also contain theories that do not correspond, to our knowledge, to already studied theories, even up to field redefinition.
\end{abstract}

\section{INTRODUCTION}

Scalar tensor theories play a prominent role in theories of modified gravity. As ever more sophisticated models have been considered, special attention was lately devoted to scalar tensor Lagrangians that contain second order derivatives of a scalar field. A crucial requirement for such theories is the absence of the so-called Ostrogradski ghost, in order to avoid disastrous instabilities [1]. It has been thought for a long time that the absence of an Ostrogradski ghost demands the Euler-Lagrange equations to be at most second-order, which explains why the literature has been mostly limited to the study of Horndeski's theories [2, 3] until recently. But the discovery of viable theories "beyond Horndeski" [4 6], i.e. possessing Euler-Lagrange equations for the metric and scalar field whose order is higher than two, has challenged this preconception.

A higher order scalar tensor theory generically contains four degrees of freedom, including the Ostrogradski ghost. As we proposed in [7], a systematic way to identify scalar-tensor theories that contain only three degrees of freedom is to consider Lagrangians that are degenerate, in a generalized sense involving the coupling between the metric and the scalar field. From the Hamiltonian point of view, this degeneracy implies the existence of phase space constraints, in addition to the usual Hamiltonian and momentum constraints due to diffeomorphism invariance, and explains why one degree of freedom is eliminated, even if the equations of motion are higher order. A detailed Hamiltonian analysis has confirmed the direct link between this degeneracy and the elimination of the Ostrogradski ghost [8].

The degeneracy criterium, which provides a powerful and simple method to identify viable

*Electronic address: jibrilbenachour@gmail.com

${ }^{\dagger}$ Electronic address: langlois@apc.univ-paris7.fr

${ }^{\ddagger}$ Electronic address: karim.noui@lmpt.univ-tours.fr 
theories, was used in [7] to find all scalar tensor theories based on a Lagrangian quadratic in second order derivatives of a scalar field, together with a term proportional to the scalar curvature. Within these degenerate higher order scalar tensor (DHOST) theories, we recovered, as particular cases, the (quadratic) Horndeski Lagrangian $L_{4}^{\mathrm{H}}$ as well as its extension $L_{4}^{\mathrm{bH}}$ introduced in [5, 6] (see section II for an explicit definition of these Lagrangians). We also considered the quintic extension beyond Horndeski of [5], $L_{5}^{\mathrm{bH}}$, which is degenerate by itself or combined with $L_{4}^{\mathrm{bH}}$ but not with an arbitrary $L_{4}^{\mathrm{H}}$. By using the same degeneracy argument, the combinations involving $L_{5}^{\mathrm{H}}$ too were studied in [9]. In particular, the results of [7, 9] show that only specific combinations of Horndeski Lagrangians with their quartic and quintic extensions beyond Horndeski are viable and they coincide with the combinations obtained in [6] via disformal transformation of Horndeski.

The goal of the present work is to examine in more detail all the quadratic DHOST theories of [7] and investigate whether they can be related, or not, to already known theories via generalized disformal transformations [10], i.e. redefinitions of the metric of the form

$$
\tilde{g}_{\mu \nu}=A(X, \phi) g_{\mu \nu}+B(X, \phi) \nabla_{\mu} \phi \nabla_{\nu} \phi,
$$

where $X \equiv g^{\mu \nu} \nabla_{\mu} \phi \nabla_{\nu} \phi$. Several results concerning the disformal transformations of Horndeski theories have already been established in previous works. It was shown, in [11], that Horndeski theories transform into themselves under special disformal transformations where $A$ and $B$ depend on $\phi$ only, not on $X$. The general disformal transformation of the Einstein-Hilbert Lagrangian was computed in [4], providing the first example of theory "beyond Horndeski", i.e. a ghost-free theory with higher order Euler-Lagrange equations of motion. In [6], it was shown that disformal transformations of Horndeski theories with $A=1$ lead to the extensions beyond Horndeski proposed in [5]. The $X$-dependent disformal transformations have also been studied recently in several papers (see e.g. [13 17], and [18] for scalar tensor theories that explicitly break spacetime covariance [19].)

In this work, we present the general disformal transformation of all quadratic DHOST theories identified in [7]. This is useful to show that the three main classes of theories, as well as the few subclasses within each, are stable under disformal transformations. Part of our results coincides with the conclusions of [12], which also studies the theories of [7] and especially the class of theories related to (quadratic) Horndeski via disformal transformations. Here, we derive the transformation laws of the arbitrary functions in the general action, which enables us to study the disformal transformations in the other classes as well.

Interestingly, the quadratic DHOST theories contain a few theories which have been well studied in the literature. Indeed, the Lagrangians that remain invariant under a field redefinition of the scalar field correspond to khronometric theories [20], which are a subset of Einstein-Aether theories [21]. Note that khronometric theories are not in general degenerate and only a subset of them appear among DHOST theories. Finally, we also discuss mimetic gravity 22] and related theories [23], which are obtained from the Einstein-Hilbert action by a disformal transformation that is not invertible (see also [4]).

Our paper is organized as follows. In the next section, we introduce the general form of the Lagrangians we will study. In section [II], we summarize the main results obtained in [7] and present the classification of quadratic DHOST theories. In section IV we derive the general disformal transformation of any quadratic DHOST Lagrangian. This enables us to show that all classes are stable under these transformations. In section $\mathrm{V}$, we consider the theories related to Horndeski. Other classes are analysed in the subsequent section. In section VII, we discuss the degenerate khronometric theories as well as mimetic theories. We conclude in the final section. 


\section{SCALAR TENSOR THEORIES}

\section{A. The action}

In this work, we consider scalar-tensor theories whose dynamics is governed by an action of the general form

$$
S=S_{g}+S_{\phi}
$$

where the first contribution involves the Ricci scalar $R$ of the metric $g_{\mu \nu}$,

$$
S_{g} \equiv \int d^{4} x \sqrt{-g} f(\phi, X) R
$$

and the second contribution depends quadratically on the second derivatives of the scalar field $\phi$

$$
S_{\phi} \equiv \int d^{4} x \sqrt{-g} C^{\mu \nu, \rho \sigma} \nabla_{\mu} \nabla_{\nu} \phi \nabla_{\rho} \nabla_{\sigma} \phi
$$

$C^{\mu \nu, \rho \sigma}$ being an arbitrary tensor that depends only on $\phi$ and $\nabla_{\mu} \phi$. Note that $S_{g}$ reduces to the familiar Einstein-Hilbert action when the function $f$ is constant.

We stress that our analysis is also valid if we add to the above action extra contributions that depend at most linearly on $\phi_{\mu \nu}$, i.e. of the form

$$
S_{\text {other }}=\int d^{4} x \sqrt{-g}\left\{P(\phi, X)+Q_{1}(\phi, X) g^{\mu \nu} \phi_{\mu \nu}+Q_{2}(\phi, X) \phi^{\mu} \phi_{\mu \nu} \phi^{\nu}\right\},
$$

where we have used the compact notation $\phi_{\mu} \equiv \nabla_{\mu} \phi$ and $\phi_{\mu \nu} \equiv \nabla_{\mu} \nabla_{\nu} \phi$. These additional contributions do not modify the degeneracy conditions derived in [7], which will be summarized in the next section. For simplicity, we will not include these terms explicitly in our study but one should keep in mind that they can be present.

Without loss of generality, we require the tensor $C^{\mu \nu, \rho \sigma}$ in (2.3) to satisfy the index symmetries

$$
C^{\mu \nu, \rho \sigma}=C^{\nu \mu, \rho \sigma}=C^{\mu \nu, \sigma \rho}=C^{\rho \sigma, \mu \nu},
$$

which implies that the most general form of this tensor is

$$
\begin{aligned}
C^{\mu \nu, \rho \sigma}=\frac{1}{2} \alpha_{1}\left(g^{\mu \rho} g^{\nu \sigma}+g^{\mu \sigma} g^{\nu \rho}\right)+\alpha_{2} g^{\mu \nu} g^{\rho \sigma}+\frac{1}{2} \alpha_{3}\left(\phi^{\mu} \phi^{\nu} g^{\rho \sigma}+\phi^{\rho} \phi^{\sigma} g^{\mu \nu}\right) \\
+\frac{1}{4} \alpha_{4}\left(\phi^{\mu} \phi^{\rho} g^{\nu \sigma}+\phi^{\nu} \phi^{\rho} g^{\mu \sigma}+\phi^{\mu} \phi^{\sigma} g^{\nu \rho}+\phi^{\nu} \phi^{\sigma} g^{\mu \rho}\right)+\alpha_{5} \phi^{\mu} \phi^{\nu} \phi^{\rho} \phi^{\sigma}
\end{aligned}
$$

where the $\alpha_{I}$ are five arbitrary functions of $\phi$ and $X$. Defining the five elementary Lagrangians quadratic in second derivatives

$$
\begin{aligned}
& L_{1}^{\phi} \equiv \phi^{\mu \nu} \phi_{\mu \nu}, \quad L_{2}^{\phi} \equiv\left(\phi_{\mu}^{\mu}\right)^{2}, \quad L_{3}^{\phi} \equiv \phi_{\mu}^{\mu} \phi^{\rho} \phi_{\rho \sigma} \phi^{\sigma}, \\
& L_{4}^{\phi} \equiv \phi^{\mu} \phi_{\mu \nu} \phi^{\nu \rho} \phi_{\rho}, \quad L_{5}^{\phi} \equiv\left(\phi^{\rho} \phi_{\rho \sigma} \phi^{\sigma}\right)^{2},
\end{aligned}
$$

the action $S_{\phi}$ in $(2.3)$ now reads

$$
S_{\phi}=\int d^{4} x \sqrt{-g}\left(\alpha_{1} L_{1}^{\phi}+\alpha_{2} L_{2}^{\phi}+\alpha_{3} L_{3}^{\phi}+\alpha_{4} L_{4}^{\phi}+\alpha_{5} L_{5}^{\phi}\right) \equiv \int d^{4} x \sqrt{-g} \alpha_{I} L_{I}^{\phi},
$$

where the summation over the index $I(I=1, \ldots, 5)$ is implicit in the last expression. 


\section{B. Other curvature terms}

It is not difficult to see that the general action (2.1) also includes terms of the form

$$
S_{\text {Ricci }} \equiv \int d^{4} x \sqrt{-g} h(\phi, X) R_{\mu \nu} \phi^{\mu} \phi^{\nu},
$$

where $h$ is an arbitrary function. Indeed, using the definition of the Ricci tensor and the properties of the Riemann tensor, one can write

$$
\begin{aligned}
\phi^{\mu} R_{\mu \nu} \phi^{\nu} & =-\phi^{\mu} g^{\rho \sigma} R_{\rho \mu \nu \sigma} \phi^{\nu}=-\phi^{\mu} g^{\rho \sigma}\left(\nabla_{\rho} \nabla_{\mu}-\nabla_{\mu} \nabla_{\rho}\right) \phi_{\sigma} \\
& =-\phi^{\mu} \nabla_{\mu} \nabla_{\nu} \phi^{\nu}+\phi^{\mu} \nabla_{\nu} \nabla_{\mu} \phi^{\nu}
\end{aligned}
$$

Substituting this into the action (2.9), one gets, after integration by parts,

$$
S_{\text {Ricci }} \equiv \int d^{4} x \sqrt{-g}\left\{-h\left(L_{1}^{\phi}-L_{2}^{\phi}\right)+2 h_{X}\left(L_{3}^{\phi}-L_{4}^{\phi}\right)+h_{\phi}\left(X \phi_{\mu}^{\mu}-\phi^{\mu} \phi_{\mu \nu} \phi^{\nu}\right)\right\}
$$

where the contribution proportional to $h_{\phi}$ is of the form (2.4).

\section{Particular cases}

The theories (2.1) include as a particular case the quartic Horndeski term

$$
L_{4}^{\mathrm{H}}=G_{4}(\phi, X) R-2 G_{4, X}(\phi, X)\left(\square \phi^{2}-\phi^{\mu \nu} \phi_{\mu \nu}\right),
$$

which corresponds to (2.2) and (2.8) with

$$
f=G_{4}, \quad \alpha_{1}=-\alpha_{2}=2 G_{4, X}, \quad \alpha_{3}=\alpha_{4}=\alpha_{5}=0 .
$$

The action (2.1) also includes the extension beyond Horndeski introduced in [5], which can be written as

$$
L_{4}^{\mathrm{bH}}=F_{4}(\phi, X) \epsilon_{\sigma}^{\mu \nu \rho} \epsilon^{\mu^{\prime} \nu^{\prime} \rho^{\prime} \sigma} \phi_{\mu} \phi_{\mu^{\prime}} \phi_{\nu \nu^{\prime}} \phi_{\rho \rho^{\prime}}
$$

This is of the form (2.8) with

$$
\alpha_{1}=-\alpha_{2}=X F_{4}, \quad \alpha_{3}=-\alpha_{4}=2 F_{4}, \quad \alpha_{5}=0 .
$$

Of course, any combination of $L_{4}^{\mathrm{H}}$ and $L_{4}^{\mathrm{bH}}$ is also among the theories (2.1).

\section{CLASSIFICATION OF DEGENERATE THEORIES}

In this section, we summarize the main results obtained in [7], as well as some additional elements derived in [8], and present all the quadratic DHOST theories, i.e. all the theories of the form (2.1) which are degenerate. 


\section{A. Degeneracy conditions}

In order to study the degeneracy of (2.1), it is useful to introduce the auxiliary field $A_{\mu} \equiv \nabla_{\mu} \phi$. For an arbitrary foliation of spacetime by spacelike hypersurfaces $\Sigma(t)$, endowed with spatial metric $h_{i j}$, the metric in ADM form reads

$$
d s^{2}=-N^{2} d t^{2}+h_{i j}\left(d x^{i}+N^{i} d t\right)\left(d x^{j}+N^{j} d t\right),
$$

where $N$ is the lapse and $N^{i}$ the shift vector. The $(3+1)$ decomposition of the action (2.1) leads to a kinetic term of the form [7]

$$
S_{\text {kin }}=\int d t d^{3} x N \sqrt{h}\left[\frac{1}{N^{2}} \mathcal{A} A_{*}^{2}+\frac{2}{N} \mathcal{B}^{i j} A_{*} K_{i j}+\mathcal{K}^{i j k l} K_{i j} K_{k l}\right],
$$

where we have introduced the quantity

$$
A_{*} \equiv \frac{1}{N}\left(A_{0}-N^{i} A_{i}\right)
$$

and the extrinsic curvature tensor

$$
K_{i j} \equiv \frac{1}{2 N}\left(\dot{h}_{i j}-D_{i} N_{j}-D_{j} N_{i}\right) .
$$

The coefficients that appear in (3.2) depend on the six arbitrary functions $f$ and $\alpha_{I}$ of (2.1). They are explicitly given by [7, 8]

$$
\begin{aligned}
\mathcal{A}= & \alpha_{1}+\alpha_{2}-\left(\alpha_{3}+\alpha_{4}\right) A_{*}^{2}+\alpha_{5} A_{*}^{4}, \quad \mathcal{B}^{i j}=\beta_{1} h^{i j}+\beta_{2} \hat{A}^{i} \hat{A}^{j}, \\
\mathcal{K}^{i j, k l}= & \kappa_{1} h^{i(k} h^{l) j}+\kappa_{2} h^{i j} h^{k l}+\frac{1}{2} \kappa_{3}\left(\hat{A}^{i} \hat{A}^{j} h^{k l}+\hat{A}^{k} \hat{A}^{l} h^{i j}\right) \\
& +\frac{1}{2} \kappa_{4}\left(\hat{A}^{i} \hat{A}^{(k} h^{l) j}+\hat{A}^{j} \hat{A}^{(k} h^{l) i}\right)+\kappa_{5} \hat{A}^{i} \hat{A}^{j} \hat{A}^{k} \hat{A}^{l},
\end{aligned}
$$

with

$$
\begin{aligned}
& \beta_{1}=\frac{A_{*}}{2}\left(2 \alpha_{2}-\alpha_{3} A_{*}^{2}+4 f_{, X}\right), \quad \beta_{2}=\frac{A_{*}}{2}\left(2 \alpha_{5} A_{*}^{2}-\alpha_{3}-2 \alpha_{4}\right), \\
& \kappa_{1}=\alpha_{1} A_{*}^{2}+f, \kappa_{2}=\alpha_{2} A_{*}^{2}-f, \kappa_{3}=-\alpha_{3} A_{*}^{2}+4 f_{, X}, \kappa_{4}=-2 \alpha_{1}, \kappa_{5}=\alpha_{5} A_{*}^{2}-\alpha_{4} .
\end{aligned}
$$

The three-dimensional vector $\hat{A}^{i}$ is defined by $\hat{A}_{i} \equiv A_{i}$ and $\hat{A}^{i} \equiv h^{i j} \hat{A}_{j}$.

By choosing an appropriate basis of the six-dimensional vector space of symmetric $3 \times 3$ matrices, where the $K_{i j}$ take their values, the kinetic matrix associated with (3.2) can be written as a $7 \times 7$ block diagonal symmetric matrix of the form [8]

$$
\left(\begin{array}{cc}
\mathcal{M} & \mathbf{0} \\
\mathbf{0} & \mathcal{D}
\end{array}\right)
$$

with the $3 \times 3$ matrix

$$
\mathcal{M} \equiv\left(\begin{array}{ccc}
\mathcal{A} & \frac{1}{2}\left(\beta_{1}+\hat{A}^{2} \beta_{2}\right) & \frac{1}{\sqrt{2}} \beta_{1} \\
\frac{1}{2}\left(\beta_{1}+\hat{A}^{2} \beta_{2}\right) & \kappa_{1}+\kappa_{2}+\hat{A}^{2}\left(\kappa_{3}+\kappa_{4}\right)+\left(\hat{A}^{2}\right)^{2} \kappa_{5} & \sqrt{2}\left(\kappa_{2}+\frac{1}{2} \hat{A}^{2} \kappa_{3}\right) \\
\frac{1}{\sqrt{2}} \beta_{1} & \sqrt{2}\left(\kappa_{2}+\frac{1}{2} \hat{A}^{2} \kappa_{3}\right) & \kappa_{1}+2 \kappa_{2}
\end{array}\right)
$$

and the diagonal matrix

$$
\mathcal{D}=\operatorname{Diag}\left[\kappa_{1}, \kappa_{1}, \kappa_{1}+\frac{1}{2} \hat{A}^{2} \kappa_{4}, \kappa_{1}+\frac{1}{2} \hat{A}^{2} \kappa_{4}\right] .
$$


The coefficients in the first line (or first row) of $\mathcal{M}$ describe the kinetic terms associated with the scalar field related variable $A_{*}$, including its mixing with the metric sector. As for the metric sector alone, it is described by the right lower $2 \times 2$ submatrix of $\mathcal{M}$, which we will call $\mathcal{M}_{K}$, together with $\mathcal{D}$. As our goal is to eliminate the extra degree of freedom due to the higher derivatives of the scalar field, we are looking for a degeneracy of the kinetic matrix that arises from the scalar sector. As a consequence, we will be interested in theories such that $\mathcal{M}$ is degenerate, while $\mathcal{M}_{K}$ and $\mathcal{D}$ remain nondegenerate in order to preserve the usual tensor structure of gravity.

Requiring the determinant of the matrix $\mathcal{M}$ to vanish ${ }^{1}$ yields an expression of the form

$$
D_{0}(X)+D_{1}(X) A_{*}^{2}+D_{2}(X) A_{*}^{4}=0,
$$

where we have substituted the expressions (3.5)-(3.8) into (3.10) and replaced all $\hat{A}^{2}$ by $X+A_{*}^{2}$. The functions $D_{0}, D_{1}$ and $D_{2}$ depend on the six arbitrary functions $\tilde{f}$ and $\alpha_{I}$ of the initial Lagrangian:

$$
\begin{aligned}
& D_{0}(X) \equiv-4\left(\alpha_{1}+\alpha_{2}\right)\left[X f\left(2 \alpha_{1}+X \alpha_{4}+4 f_{X}\right)-2 f^{2}-8 X^{2} f_{X}^{2}\right], \\
& D_{1}(X) \equiv 4\left[X^{2} \alpha_{1}\left(\alpha_{1}+3 \alpha_{2}\right)-2 f^{2}-4 X f \alpha_{2}\right] \alpha_{4}+4 X^{2} f\left(\alpha_{1}+\alpha_{2}\right) \alpha_{5} \\
& +8 X \alpha_{1}^{3}-4\left(f+4 X f_{X}-6 X \alpha_{2}\right) \alpha_{1}^{2}-16\left(f+5 X f_{X}\right) \alpha_{1} \alpha_{2}+4 X\left(3 f-4 X f_{X}\right) \alpha_{1} \alpha_{3} \\
& -X^{2} f \alpha_{3}^{2}+32 f_{X}\left(f+2 X f_{X}\right) \alpha_{2}-16 f f_{X} \alpha_{1}-8 f\left(f-X f_{X}\right) \alpha_{3}+48 f f_{X}^{2} \text {, } \\
& D_{2}(X) \equiv 4\left[2 f^{2}+4 X f \alpha_{2}-X^{2} \alpha_{1}\left(\alpha_{1}+3 \alpha_{2}\right)\right] \alpha_{5}+4 \alpha_{1}^{3}+4\left(2 \alpha_{2}-X \alpha_{3}-4 f_{X}\right) \alpha_{1}^{2}+3 X^{2} \alpha_{1} \alpha_{3}^{2} \\
& -4 X f \alpha_{3}^{2}+8\left(f+X f_{X}\right) \alpha_{1} \alpha_{3}-32 f_{X} \alpha_{1} \alpha_{2}+16 f_{X}^{2} \alpha_{1}+32 f_{X}^{2} \alpha_{2}-16 f f_{X} \alpha_{3} \text {. }
\end{aligned}
$$

Since the determinant must vanish for any value of $A_{*}$, we deduce that degenerate theories are characterized by the three conditions

$$
D_{0}(X)=0, \quad D_{1}(X)=0, \quad D_{2}(X)=0 .
$$

By solving these three conditions, one can determine and classify all DHOST theories, as discussed in [7].

\section{B. Degenerate theories}

The condition $D_{0}(X)=0$ is the simplest of all three and allows to distinguish several classes of theories. Indeed, $D_{0}$ can vanish either if $\alpha_{1}+\alpha_{2}=0$, which defines our first class of solutions, or if the term between brackets in (3.13) vanishes, which defines our second class, as well as our third class corresponding to the special case where $f=0$.

$$
\text { 1. Class } I\left(\alpha_{1}+\alpha_{2}=0\right)
$$

This class is characterized by the property

$$
\alpha_{1}=-\alpha_{2}
$$

\footnotetext{
${ }^{1}$ Note that we have not used the same matrix in 7] but another, non symmetric, matrix constructed by solving for null eigenvectors of the kinetic matrix. The two methods are obviously equivalent.
} 
One can then use the conditions $D_{1}(X)=0$ and $D_{2}(X)=0$ to express, respectively, $\alpha_{4}$ and $\alpha_{5}$ in terms of $\alpha_{2}$ and $\alpha_{3}$, provided $f+X \alpha_{2} \neq 0$. This defines the subclass Ia, characterized by

$$
\begin{gathered}
\alpha_{4}=\frac{1}{8\left(f+X \alpha_{2}\right)^{2}}\left[16 X \alpha_{2}^{3}+4\left(3 f+16 X f_{X}\right) \alpha_{2}^{2}+\left(16 X^{2} f_{X}-12 X f\right) \alpha_{3} \alpha_{2}-X^{2} f \alpha_{3}^{2}\right. \\
\left.+16 f_{X}\left(3 f+4 X f_{X}\right) \alpha_{2}+8 f\left(X f_{X}-f\right) \alpha_{3}+48 f f_{X}^{2}\right]
\end{gathered}
$$

and

$$
\alpha_{5}=\frac{\left(4 f_{X}+2 \alpha_{2}+X \alpha_{3}\right)\left(-2 \alpha_{2}^{2}+3 X \alpha_{2} \alpha_{3}-4 f_{X} \alpha_{2}+4 f \alpha_{3}\right)}{8\left(f+X \alpha_{2}\right)^{2}} .
$$

Degenerate theories in class Ia thus depend on three arbitrary functions $\alpha_{2}, \alpha_{3}$ and $f$.

In the special case $f+X \alpha_{2}=0$, we find another subclass of solutions characterized by

$$
\alpha_{1}=-\alpha_{2}=\frac{f}{X}, \quad \alpha_{3}=\frac{2}{X^{2}}\left(f-2 X f_{X}\right), \quad \text { (Class Ib), }
$$

where $f, \alpha_{4}$ and $\alpha_{5}$ are arbitrary functions. In the following, we will not explore this class much further because the metric sector is degenerate. Indeed, the last two eigenvalues of $\mathcal{D}$, which are equal to $f-\alpha_{1} X$, vanish in this case.

\section{Class II}

The condition $D_{0}(X)=0$ can also be satisfied if

$$
X f\left(2 \alpha_{1}+X \alpha_{4}+4 f_{X}\right)-2 f^{2}-8 X^{2} f_{X}^{2}=0 .
$$

We can then proceed as previously by solving $D_{1}(X)=0$ and $D_{2}(X)=0$ to express $\alpha_{4}$ and $\alpha_{5}$ in terms of the three other functions. Substituting the obtained expression for $\alpha_{4}$ into the condition (3.21), one finally gets

$$
\left(X \alpha_{1}-f\right)\left[\left(4 f^{2}+X f\left(8 \alpha_{2}+2 \alpha_{1}+X \alpha_{3}-4 f_{X}\right)-4 X^{2} f_{X}\left(\alpha_{1}+3 \alpha_{2}\right)\right]=0 .\right.
$$

Assuming that $f-X \alpha_{1} \neq 0$, this leads to the expressions

$$
\begin{aligned}
& \alpha_{3}=\frac{1}{X^{2} f}\left[-4 f\left(f-X f_{X}\right)-2 X\left(f-2 X f_{X}\right) \alpha_{1}+4 X\left(-2 f+3 X f_{X}\right)\right], \\
& \alpha_{4}=\frac{2}{X^{2} f}\left[f^{2}-2 f X f_{X}+4 X^{2} f_{X}^{2}-X f \alpha_{1}\right], \\
& \alpha_{5}=\frac{2}{f^{2} X^{3}}\left[4 f\left(f^{2}-3 f X f_{X}+2 X^{2} f_{X}^{2}\right)+\left(3 X f^{2}-8 X^{2} f f_{X}+6 X^{3} f_{X}^{2}\right) \alpha_{1}\right. \\
& \left.\quad+2 X\left(2 f-3 X f_{X}\right)^{2} \alpha_{2}\right],
\end{aligned}
$$

while $\tilde{f}, \alpha_{1}$ and $\alpha_{2}$ are arbitrary. This describes our class IIa, characterized by three arbitrary functions.

The case $f=X \alpha_{1}$ defines another class, similar to class Ib, which we will call class IIb, described by

$$
\begin{aligned}
& \alpha_{1}=\frac{f}{X}, \quad \alpha_{4}=4 f_{X}\left(2 \frac{f_{X}}{f}-\frac{1}{X}\right) \\
& \begin{aligned}
\alpha_{5}=\frac{1}{4 X^{3} f\left(f+X \alpha_{2}\right)}[ & 8 X\left(4 X f_{X} f-f^{2}-4 X^{2} f_{X}^{2}\right) \alpha_{2}+X f\left(8 X^{2} f_{X}+X^{3} \alpha_{3}-4 f\right) \alpha_{3} \\
& \left.+4\left(X f_{X} f^{2}-2 X^{3} f_{X}^{3}+2 X^{2} f_{X}^{2} f-f^{3}\right)\right],
\end{aligned}
\end{aligned}
$$

where $\alpha_{2}$ and $\alpha_{3}$ are arbitrary functions. Like class $\mathrm{Ib}$, the metric sector is degenerate for these theories and we will not consider them further in the following. 


\section{Class III $(f=0)$}

Finally, we devote a special class to the case $f=0$, which also leads automatically to $D_{0}=0$. Using $D_{1}=0$ and $D_{2}=0$ to determine $\alpha_{4}$ and $\alpha_{5}$, one gets

$$
\alpha_{4}=-\frac{2}{X} \alpha_{1}, \quad \alpha_{5}=\frac{4 \alpha_{1}^{2}+8 \alpha_{1} \alpha_{2}-4 \alpha_{1} \alpha_{3} X+3 \alpha_{3}^{2} X^{2}}{4 X^{2}\left(\alpha_{1}+3 \alpha_{2}\right)} \quad \text { (class IIIa) },
$$

while $\alpha_{1}, \alpha_{2}$ and $\alpha_{3}$ are arbitrary, provided $\alpha_{1}+3 \alpha_{2} \neq 0$. This defines our class IIIa. Note that the intersection of IIIa with the class Ia is described by

$$
\alpha_{2}=-\alpha_{1}, \quad \alpha_{4}=-\frac{2}{X} \alpha_{1}, \quad \alpha_{5}=\frac{\left(2 \alpha_{1}-X \alpha_{3}\right)\left(2 \alpha_{1}+3 X \alpha_{3}\right)}{8 X^{2} \alpha_{1}}, \quad(\text { IIIa } \cap \text { Ia })
$$

which depends on two arbitrary functions, $\alpha_{1}$ and $\alpha_{3}$, and includes the Lagrangian $L_{4}^{\text {bh }}$ (for which $\left.\alpha_{1} / X=\alpha_{3} / 2=F_{4}\right)$.

The case $\alpha_{1}+3 \alpha_{2}=0$ yields another subclass,

$$
f=0, \quad \alpha_{1}=\frac{3}{2} X \alpha_{3}, \quad \alpha_{2}=-\frac{X}{2} \alpha_{3} \quad(\text { class IIIb }),
$$

which in general leads to a degenerate metric sector. Another special case corresponds to the class

$$
f=0, \quad \alpha_{1}=0, \quad \text { (class IIIc) }
$$

which depends on four arbitrary functions. Since $f-\alpha_{1} X=0$, this class is also degenerate in the metric sector.

\section{Degeneracy of the scalar sector alone}

Among all the degenerate theories that we have listed above, it is not difficult to identify the theories that remain degenerate even when the metric becomes nondynamical, as noted in [7]. In this limit, only the kinetic term for $A_{*}$ is relevant and the degeneracy of the scalar sector alone thus requires $\mathcal{A}=0$, which imposes simultaneously the three constraints

$$
\alpha_{1}+\alpha_{2}=0, \quad \alpha_{3}+\alpha_{4}=0, \quad \alpha_{5}=0 .
$$

The first condition implies that the theories satisfying these conditions belong to class I. Ignoring class Ib, whose metric sector is degenerate, we turn to class Ia. For theories satisfying (3.32), the functions $\alpha_{2}$ and $\alpha_{3}$ are no longer independent, but related by

$$
4 f_{X}+2 \alpha_{2}+X \alpha_{3}=0
$$

This means that the condition $\mathcal{A}=0$ restricts the degenerate theories to a subclass that depends on two arbitrary functions only. It is easy to see that this family of theories in fact coincides with the sum of $L_{4}^{\mathrm{H}}$ and $L_{4}^{\mathrm{bH}}$, upon using the identification

$$
f=G_{4}, \quad \alpha_{1}=-\alpha_{2}=2 G_{4 X}+X F_{4}, \quad \alpha_{3}=-\alpha_{4}=2 F_{4} .
$$

This implies that the quartic Lagrangian $L_{4}=L_{4}^{\mathrm{H}}+L_{4}^{\mathrm{bH}}$ represents the most general theories that are degenerate when the metric is nondynamical (with a nondegenerate metric sector). 


\section{DISFORMAL TRANSFORMATIONS}

We now study the effect of conformal-disformal transformations, or generalized disformal transformations, introduced in [10], in which the "disformed" metric $\tilde{g}_{\mu \nu}$ is expressed in terms of $g_{\mu \nu}$ and $\phi$ as

$$
\tilde{g}_{\mu \nu}=A(X, \phi) g_{\mu \nu}+B(X, \phi) \phi_{\mu} \phi_{\nu} .
$$

Via this transformation, any action $\tilde{S}$ given as a functional of $\tilde{g}_{\mu \nu}$ and $\phi$ induces a new action $S$ for $g_{\mu \nu}$ and $\phi$, when one substitutes the above expression for $\tilde{g}_{\mu \nu}$ in $\tilde{S}$ :

$$
S\left[\phi, g_{\mu \nu}\right] \equiv \tilde{S}\left[\phi, \tilde{g}_{\mu \nu}=A g_{\mu \nu}+B \phi_{\mu} \phi_{\nu}\right] .
$$

We will say that the actions $S$ and $\tilde{S}$ are related by the disformal transformation (4.1).

Starting from an action $\tilde{S}$ of the form (2.1),

$$
\tilde{S}=\tilde{S}_{g}+\tilde{S}_{\phi}=\int d^{4} x \sqrt{-\tilde{g}}\left[\tilde{f} \tilde{R}+\tilde{\alpha}_{I} \tilde{L}_{I}^{\phi}\right]
$$

we show below that the action $S$, related to $\tilde{S}$ via a disformal transformation, is also of the form (2.1), up to terms of the form (2.4), and we compute explicitly the relations between the functions that appear in the two Lagrangians. Interestingly, if the disformal transformation is invertible, in the sense that the metric $g_{\mu \nu}$ can be expressed in terms of $\tilde{g}_{\mu \nu}$, then the number of degrees of freedom associated with $S$ and $\tilde{S}$ should be the same. One thus expects that the disformal transformations of all the degenerate theories described in the previous section are also degenerate. We will also discuss the special case where the transformation is non invertible in subsectionVIIB.

\section{A. Relations between the two metrics and their covariant derivatives}

In order to write explicitly the above action in terms of $g_{\mu \nu}$ and $\phi$, we will need the expression of the inverse metric

$$
\tilde{g}^{\mu \nu}=A^{-1}\left(g^{\mu \nu}-\frac{B}{A+B X} \nabla^{\mu} \phi \nabla^{\nu} \phi\right)
$$

Contracting this relation with $\phi_{\mu} \phi_{\nu}$ gives $\tilde{X}$ as a function of $X$ :

$$
\tilde{X}=\frac{X}{A+B X}
$$

It is also useful to introduce the ratio

$$
\mathcal{J}_{g} \equiv \frac{\sqrt{-\tilde{g}}}{\sqrt{-g}}=A^{3 / 2} \sqrt{A+B X} .
$$

The difference between the two covariant derivatives $\tilde{\nabla}$ and $\nabla$, associated respectively to the two metrics $\tilde{g}_{\mu \nu}$ and $g_{\mu \nu}$, is fully characterized by the difference of their respective Christoffel symbols,

$$
C_{\mu \nu}^{\lambda} \equiv \tilde{\Gamma}_{\mu \nu}^{\lambda}-\Gamma_{\mu \nu}^{\lambda}
$$

which defines a tensor. In particular, the relation between the respective second order covariant derivatives of $\phi$ reads

$$
\tilde{\nabla}_{\mu} \tilde{\nabla}_{\nu} \phi=\nabla_{\mu} \nabla_{\nu} \phi-C_{\mu \nu}^{\lambda} \phi_{\lambda}
$$


The explicit expression for $C_{\mu \nu}^{\lambda}$ is given by

$$
\begin{aligned}
C_{\mu \nu}^{\lambda}= & \frac{A_{X}}{A}\left[2 \delta_{(\mu}^{\lambda} \phi_{\nu) \sigma} \phi^{\sigma}-\phi^{\lambda \sigma} \phi_{\sigma} g_{\mu \nu}+\frac{B}{A+B X}\left(-2 \phi^{\lambda} \phi_{(\mu} \phi_{\nu) \sigma} \phi^{\sigma}+\phi^{\lambda} \phi^{\rho} \phi_{\rho \sigma} \phi^{\sigma} g_{\mu \nu}\right)\right] \\
& +B_{X}\left[-\frac{1}{A} \phi_{\mu} \phi_{\nu} \phi^{\lambda \sigma} \phi_{\sigma}+\frac{1}{A+B X}\left(2 \phi^{\lambda} \phi_{(\mu} \phi_{\nu) \sigma} \phi^{\sigma}+\frac{B}{A} \phi^{\rho} \phi_{\rho \sigma} \phi^{\sigma} \phi^{\lambda} \phi_{\mu} \phi_{\nu}\right)\right]+\frac{B}{A+B X} \phi^{\lambda} \phi_{\mu \nu} \\
& +\frac{A_{\phi}}{2 A}\left[\delta_{\mu}^{\lambda} \phi_{\nu}+\delta_{\nu}^{\lambda} \phi_{\mu}-\frac{1}{A+B X}\left(A \phi^{\lambda} g_{\mu \nu}+2 B \phi^{\lambda} \phi_{\mu} \phi_{\nu}\right)\right]+\frac{B_{\phi}}{2(A+B X)} \phi^{\lambda} \phi_{\mu} \phi_{\nu} .
\end{aligned}
$$

The last line does not depend on second derivatives of $\phi$. As we will see, this implies that the terms in $A_{\phi}$ and $B_{\phi}$ appear only in the transformed action as terms of the form (2.4), which we will not compute explicitly.

\section{B. Curvature term}

Let us first concentrate, in $\tilde{S}$, on the term depending on the Ricci scalar of $\tilde{g}_{\mu \nu}$. Following the derivation presented in [4], the Ricci scalar $\tilde{R}$ can be written in terms of the tensor $C_{\mu \nu}^{\lambda}$ and of the metric $g_{\mu \nu}$, according to the expression

$$
\tilde{R} \equiv \tilde{g}^{\mu \nu} \tilde{R}_{\mu \nu}=A^{-1}\left(g^{\mu \nu}-\frac{B}{A+B X} \phi^{\mu} \phi^{\nu}\right)\left(R_{\mu \nu}+C_{\mu \rho}^{\sigma} C_{\nu \sigma}^{\rho}-C_{\mu \nu}^{\rho} C_{\rho \sigma}^{\sigma}\right)+\tilde{\nabla}_{\rho} \xi^{\rho}
$$

with

$$
\xi^{\rho} \equiv \tilde{g}^{\mu \nu} C_{\mu \nu}^{\rho}-\tilde{g}^{\rho \mu} C_{\mu \nu}^{\nu}
$$

All the terms quadratic in $C_{\mu \nu}^{\lambda}$ can be rewritten in terms of the elementary Lagrangians $L_{I}^{\phi}$. One finds

$$
A^{-1}\left(g^{\mu \nu}-\frac{B}{A+B X} \phi^{\mu} \phi^{\nu}\right)\left(C_{\mu \rho}^{\sigma} C_{\nu \sigma}^{\rho}-C_{\mu \nu}^{\rho} C_{\rho \sigma}^{\sigma}\right)=\sum_{I} \gamma_{I} L_{I}^{\phi}+(\ldots),
$$

with

$$
\begin{aligned}
& \gamma_{1}=\gamma_{2}=0, \quad \gamma_{3}=-\frac{B\left(B X A_{X}+A\left(2 A_{X}+X B_{X}+B\right)\right)}{A^{2}(A+B X)^{2}} \\
& \gamma_{4}=\frac{\left(6 A^{2}+8 A B X+2 B^{2} X^{2}\right) A_{X}^{2}+4 A X(A+B X) A_{X} B_{X}+A^{2} B\left(B+X B_{X}\right)}{A^{3}(A+B X)^{2}} \\
& \gamma_{5}=-\frac{2 A_{X}\left(B A_{X}+2 A B_{X}\right)}{A^{3}(A+B X)}
\end{aligned}
$$

The dots in (4.12) indicate terms that are at most linear in $\phi_{\mu \nu}$, i.e. of the form (2.4), which we will not write down explicitly.

The total derivative $\tilde{\nabla}_{\rho} \xi^{\rho}$ can be ignored if the function $\tilde{f}$ multiplying the scalar curvature is a constant. Otherwise, one also needs to reexpress this term as a function of $g_{\mu \nu}$ and $\phi$. This can be done after an integration by parts so that one gets

$$
\int d^{4} x \sqrt{-\tilde{g}} \tilde{f} \tilde{\nabla}_{\mu} \xi^{\mu}=-\int d^{4} x \sqrt{-\tilde{g}} \xi^{\mu} \nabla_{\mu} \tilde{f}=-2 \int d^{4} x \sqrt{-\tilde{g}} \tilde{f}_{\tilde{X}} \tilde{X}_{X} \xi^{\mu} \phi_{\mu \nu} \phi^{\nu}+(\ldots),
$$


with

$$
\tilde{X}_{X} \equiv \frac{\partial \tilde{X}}{\partial X}=\frac{A}{(A+B X)^{2}}
$$

Since $\xi^{\mu}$ contains second derivatives of $\phi$, the scalar quantity $\xi^{\mu} \phi_{\mu \nu} \phi^{\nu}$ can be decomposed as a combination of the elementary terms $L_{I}^{\phi}$. One finds

$$
\xi^{\mu} \phi_{\mu \nu} \phi^{\nu}=\lambda_{I} L_{I}^{\phi}+(\ldots)
$$

with

$$
\begin{aligned}
& \lambda_{1}=\lambda_{2}=0, \quad \lambda_{3}=\frac{B}{A^{2}+A B X}, \\
& \lambda_{4}=-\frac{4 B X A_{X}+A\left(6 A_{X}+2 X B_{X}+B\right)}{A^{2}(A+B X)}, \quad \lambda_{5}=\frac{2\left(2 B A_{X}+A B_{X}\right)}{A^{2}(A+B X)},
\end{aligned}
$$

and the dots stand as usual for the terms at most linear in $\phi_{\mu \nu}$.

Putting everything together, one finds that the scalar curvature term yields

$$
\int d^{4} x \sqrt{-\tilde{g}} \tilde{f} \tilde{R}=\int d^{4} x \sqrt{-g} \mathcal{J}_{g}\left\{\frac{\tilde{f}}{A}\left[R-\frac{B}{A+B X} R_{\mu \nu} \phi^{\mu} \phi^{\nu}\right]+\left(\gamma_{I}-2 \tilde{X}_{X} \tilde{f}_{\tilde{X}} \lambda_{I}\right) L_{I}^{\phi}\right\}+(\ldots),
$$

where the term in $R_{\mu \nu} \phi^{\mu} \phi^{\nu}$ is of the form (2.9) with the function

$$
h=-\mathcal{J}_{g} \frac{B}{A(A+B X)} \tilde{f},
$$

and the dots correspond to terms of the form (2.4).

\section{Scalar field terms}

Let us now consider the terms quadratic in second derivatives of the scalar field. Each of the five terms in $\tilde{S}_{\phi}$ can be decomposed, after substitution of (4.1), into the five terms that appear in the final action $S_{\phi}$.

Let us illustrate this with the first term $\tilde{L}_{1}^{\phi} \equiv \tilde{\phi}_{\mu \nu} \tilde{\phi}^{\mu \nu}$, which can be decomposed as follows:

$$
\begin{aligned}
\tilde{L}_{1}^{\phi} & =\tilde{g}^{\mu \rho} \tilde{g}^{\nu \sigma} \tilde{\nabla}_{\mu} \tilde{\nabla}_{\nu} \phi \tilde{\nabla}_{\rho} \tilde{\nabla}_{\sigma} \phi \\
& =A^{-2}\left(g^{\mu \rho}-\frac{B}{A+B X} \phi^{\mu} \phi^{\rho}\right)\left(g^{\nu \sigma}-\frac{B}{A+B X} \phi^{\nu} \phi^{\sigma}\right)\left(\phi_{\mu \nu}-C_{\mu \nu}^{\lambda} \phi_{\lambda}\right)\left(\phi_{\rho \sigma}-C_{\rho \sigma}^{\tau} \phi_{\tau}\right) \\
& =\mathcal{T}_{11} L_{1}^{\phi}+\mathcal{T}_{13} L_{3}^{\phi}+\mathcal{T}_{14} L_{4}^{\phi}+\mathcal{T}_{15} L_{5}^{\phi}+(\ldots),
\end{aligned}
$$

where the coefficients are determined explicitly by substituting the expression (4.9) for $C_{\mu \nu}^{\lambda}$. Note that the term $L_{2}^{\phi}$ does not appear in the decomposition.

Proceeding similarly with all the other terms, one finally gets five similar decompositions, which can be summarized by the expression

$$
\tilde{L}_{I}^{\phi}=\mathcal{T}_{I J} L_{J}^{\phi}+(\ldots),
$$


where the summation with respect to the index $J$ is implicit. The nonvanishing coefficients $\mathcal{T}_{I J}$ are given by

$$
\begin{aligned}
& \mathcal{T}_{11}=\frac{1}{(A+B X)^{2}}, \quad \mathcal{T}_{13}=\frac{2 A_{X}}{A(A+B X)^{2}}, \quad \mathcal{T}_{14}=\frac{2\left(X\left(A_{X}+X B_{X}\right)^{2}-A\left(2\left(A_{X}+X B_{X}\right)+B\right)\right)}{A(A+B X)^{3}}, \\
& \mathcal{T}_{15}=\frac{1}{A^{2}(A+B X)^{4}}\left[2 A^{3} B_{X}+A^{2}\left(-2 X A_{X} B_{X}+2 A_{X}^{2}+B^{2}-X^{2} B_{X}^{2}+4 B X B_{X}\right)+3 B^{2} X^{2} A_{X}^{2}\right. \\
& \left.\quad-2 A B X\left(2 X A_{X} B_{X}+A_{X}\left(B-2 A_{X}\right)+X^{2} B_{X}^{2}\right)\right] \\
& \mathcal{T}_{22}=\frac{1}{(A+B X)^{2}}, \quad \mathcal{T}_{23}=-\frac{2\left(A\left(-2 A_{X}+X B_{X}+B\right)-3 B X A_{X}\right)}{A(A+B X)^{3}}, \\
& \mathcal{T}_{25}=\frac{\left(A\left(-2 A_{X}+X B_{X}+B\right)-3 B X A_{X}\right)^{2}}{A^{2}(A+B X)^{4}}, \\
& \mathcal{T}_{33}=\frac{A-X\left(A_{X}+X B_{X}\right)}{(A+B X)^{4}}, \quad \mathcal{T}_{35}=\frac{\left(A\left(-2 A_{X}+X B_{X}+B\right)-3 B X A_{X}\right)\left(A-X\left(A_{X}+X B_{X}\right)\right)}{A(A+B X)^{5}}, \\
& \mathcal{T}_{44}=\frac{\left(A-X\left(A_{X}+X B_{X}\right)\right)^{2}}{A(A+B X)^{4}}, \quad \mathcal{T}_{45}=-\frac{B\left(A-X\left(A_{X}+X B_{X}\right)\right)^{2}}{A(A+B X)^{5}}, \\
& \mathcal{T}_{55}=\frac{\left(A-X\left(A_{X}+X B_{X}\right)\right)^{2}}{(A+B X)^{6}} .
\end{aligned}
$$

It can be noticed that these coefficients form a triangular matrix.

\section{Transformation of the total action}

Collecting all the results obtained above, one can now write the functions that appear in the action $S$ in terms of the functions $\tilde{f}$ and $\tilde{\alpha}_{I}$ of $\tilde{S}$. We find

$$
\begin{aligned}
f & =\mathcal{J}_{g} A^{-1} \tilde{f} \\
\alpha_{1} & =-h+\mathcal{J}_{g} \mathcal{T}_{11} \tilde{\alpha}_{1}, \\
\alpha_{2} & =h+\mathcal{J}_{g} \mathcal{T}_{22} \tilde{\alpha}_{2} \\
\alpha_{3} & =2 h_{X}+\mathcal{J}_{g}\left[\tilde{f} \gamma_{3}-2 \tilde{X}_{X} \tilde{f}_{\tilde{X}} \lambda_{3}+\mathcal{T}_{13} \tilde{\alpha}_{1}+\mathcal{T}_{23} \tilde{\alpha}_{2}+\mathcal{T}_{33} \tilde{\alpha}_{3}\right] \\
\alpha_{4} & =-2 h_{X}+\mathcal{J}_{g}\left[\tilde{f} \gamma_{4}-2 \tilde{X}_{X} \tilde{f}_{\tilde{X}} \lambda_{4}+\mathcal{T}_{14} \tilde{\alpha}_{1}+\mathcal{T}_{44} \tilde{\alpha}_{4}\right] \\
\alpha_{5} & =\mathcal{J}_{g}\left[\tilde{f} \gamma_{5}-2 \tilde{X} \tilde{f}_{\tilde{X}} \lambda_{5}+\mathcal{T}_{15} \tilde{\alpha}_{1}+\mathcal{T}_{25} \tilde{\alpha}_{2}+\mathcal{T}_{35} \tilde{\alpha}_{5}+\mathcal{T}_{45} \tilde{\alpha}_{5}+\mathcal{T}_{55} \tilde{\alpha}_{5}\right] .
\end{aligned}
$$

By substituting all the formulas given in the previous subsections, one obtains the explicit expressions of $f$ and $\alpha_{I}$ in terms of $\tilde{f}, \tilde{\alpha}_{I}, A$ and $B$. One can verify that the $f$ and $\alpha_{I}$ satisfy the degeneracy conditions (3.13)-(3.15). In fact, it turns out that this is a very efficient way to check the expressions for $f$ and $\alpha_{I}$. A first conclusion is thus that all quadratic DHOST theories transform into quadratic DHOST theories.

For a more detailed analyis, the relations (4.27)-(4.32) in their abridged form are useful to see how the main families of DHOST theories transform. First of all, let us note that if $\tilde{f}=0$ then necessarily $f=0$. Therefore, the transformed version of theories in class III remains in class III. As a consequence of $\mathcal{T}_{11}=\mathcal{T}_{22}$, we also find the relation

$$
\alpha_{1}+\alpha_{2}=\mathcal{J}_{g} \mathcal{T}_{11}\left(\tilde{\alpha}_{1}+\tilde{\alpha}_{2}\right)
$$

which shows that the property $\alpha_{1}+\alpha_{2}=0$ (or $\alpha_{1}+\alpha_{2} \neq 0$ ) is unchanged by disformal transformations. This implies that class I, characterized by $\alpha_{1}+\alpha_{2}=0$, is stable under disformal 
transformations. Therefore all the three main classes are stable. We study more precisely the impact of disformal transformations in the next two sections.

\section{DISFORMAL TRANSFORMATIONS IN CLASS IA}

Disformal transformations for theories in class Ia have been partially studied in several previous works. In particular, it has been shown that Horndeski theories are stable under $X$-independent disformal transformations [11]. The first example of theory beyond Hordenski, i.e. with higher order equations of motion, was exhibited in [4] by considering the general disformal transformation of the Einstein-Hilbert action, which is also in class Ia. It was also shown in [6] that the extended quadratic (quintic) Lagrangian proposed in [5] can be generated from the quadratic Horndeski Lagrangian via a purely disformal transformation with $A=1$. All these examples are particular cases of disformal transformations within class Ia.

If we now consider class Ia theories such that $\tilde{f} \neq 0$, which depend on three arbitrary functions, it is natural to expect that generic theories can be "generated" from the subset of (quadratic) Horndeski theories, characterized by a single arbitrary function $\tilde{f}$, via general disformal transformations, which depend on two arbitrary functions. We can check that this is indeed the case ${ }^{2}$, by starting from the quartic Horndeski Lagrangian expressed in terms of the metric $\tilde{g}_{\mu \nu}$ and of the scalar field $\phi$,

$$
\tilde{S}\left[\phi, \tilde{g}_{\mu \nu}\right]=\int d^{4} x \sqrt{-\tilde{g}}\left\{\tilde{f}(\tilde{X}, \phi) \tilde{R}-2 \tilde{f}_{, \tilde{X}}(\tilde{X}, \phi)\left[\left(\tilde{\nabla}^{\mu} \tilde{\nabla}_{\mu} \phi\right)^{2}-\tilde{\nabla}_{\mu} \tilde{\nabla}_{\nu} \phi \tilde{\nabla}^{\mu} \tilde{\nabla}^{\nu} \phi\right]\right\} .
$$

Substituting (4.1), we obtain an action $S$ for $g_{\mu \nu}$ and $\phi$, which is characterized by the functions

$$
f=A^{1 / 2} \sqrt{A+B X} \tilde{f},
$$

and

$$
\begin{aligned}
\alpha_{1}= & -\alpha_{2}=-\frac{2 A^{3 / 2}}{(A+B X)^{3 / 2}}\left[\left(B+X B^{2}\right) \tilde{f}+2 \tilde{f}_{\tilde{X}}\right] \\
\alpha_{3}= & -\frac{2\left(B A_{X}+A B_{X}\right)}{A^{1 / 2}(A+B X)^{1 / 2}} \tilde{f}+\frac{4\left(X B_{X}-A_{X}\right) A^{1 / 2}}{(A+B X)^{3 / 2}} \\
\alpha_{4}= & \frac{2\left(A^{2} B_{X}+A A_{X}\left(2 X B_{X}+B\right)+A_{X}^{2}(3 A+B X)\right)}{A^{3 / 2}(A+B X)^{1 / 2}} \tilde{f} \\
& -\frac{4\left(-A_{X}\left(A-2 X^{2} B_{X}\right)+A X B_{X}+2 X A_{X}^{2}\right)}{A^{1 / 2}(A+B X)^{3 / 2}} \tilde{f}_{\tilde{X}} \\
\alpha_{5}= & -\frac{2 A_{X}\left(B A_{X}+2 A B_{X}\right)}{A^{3 / 2}(A+B X)^{1 / 2}} \tilde{f}+\frac{4 A_{X}\left(2 X B_{X}-A_{X}\right)}{A^{1 / 2}(A+B X)^{3 / 2}} \tilde{f}_{\tilde{X}}
\end{aligned}
$$

If one starts from a generic theory in Class I, defined by the functions $f$ and $\alpha_{I}$, it is possible to determine two functions $A$ and $B$ such that this theory is disformally related to Horndeski, as we now show. According to (5.2), the Horndeski function $\tilde{f}$ is related to $f, A$ and $B$ by

$$
\tilde{f}=A^{-1 / 2}(A+B X)^{-1 / 2} f .
$$

\footnotetext{
${ }^{2}$ The same calculation has been performed independently in the recent paper [12].
} 
Substituting this expression for $\tilde{f}$ into (5.3) yields

$$
\alpha_{1}=-\alpha_{2}=\frac{2 A f_{X}-f\left(2 A_{X}+X B_{X}\right)}{A-X A_{X}-X^{2} B_{X}}
$$

which one can solve to find $B_{X}$ in terms of $\alpha_{2}, f$ and $A$ :

$$
B_{X}=\frac{\left(2 f_{X}+\alpha_{2}\right) A-\left(2 f+X \alpha_{2}\right) A_{X}}{X\left(f+X \alpha_{2}\right)} .
$$

Substituting (5.7) and (5.9) in $\alpha_{3}$ gives

$$
\frac{A_{X}}{A}=\frac{4 f_{X}+2 \alpha_{2}+X \alpha_{3}}{4\left(f+X \alpha_{2}\right)} .
$$

Finally, by substituting successively (5.7), (5.9) and (5.10), one can rewrite $\alpha_{4}$ and $\alpha_{5}$ in terms of $f, \alpha_{2}$ and $\alpha_{3}$ and check that one recovers exactly the expressions (3.18) and (3.19). This proves that generic theories in class Ia are "generated" from the Horndeski quadratic Lagrangians via disformal transformations (4.1).

In analogy with the choice between the "Jordan frame" and "Einstein frame" for traditional scalar tensor theories, the above construction shows that theories belonging to class Ia with $f \neq 0$ can be defined either in the "Jordan frame", where the metric is minimally coupled to matter,

$$
S_{\text {total }}=\int d^{4} x \sqrt{-g}\left[f R+\alpha_{I} L_{I}^{\phi}\right]+S_{m}\left[g_{\mu \nu}, \Psi_{m}\right]
$$

or in the "Horndeski frame", where the gravitational part of the action is described by Horndeski,

$$
\tilde{S}_{\text {total }}=\int d^{4} x \sqrt{-\tilde{g}}\left\{\tilde{f} \tilde{R}-2 \tilde{f}_{, \tilde{X}}\left[(\tilde{\square} \phi)^{2}-\tilde{\phi}_{\mu \nu} \tilde{\phi}^{\mu \nu}\right]\right\}+(\ldots)+S_{m}\left[g_{\mu \nu}, \Psi_{m}\right] .
$$

In the "Horndeski frame", the matter action is nonminimally coupled, but can be expressed explicitly in terms of the "Horndeski metric" by inverting the transformation (4.1).

Note that the Einstein-Hilbert Lagrangian, with $\tilde{f}$ constant and $\tilde{\alpha}_{I}=0$, is a particular case of Horndeski. It generates, via disformal transformations, the family characterized by the expressions (5.2)-(5.6) with $\tilde{f}_{\tilde{X}}=0$. If the disformal transformation is invertible, one thus gets a family of scalar-tensor theories which are in fact general relativity in disguise and, as such, are doubly degenerate and contain only two tensor modes. Of course, one can always add another term of the form (2.4) in the action, which does not modify the quadratic part of the action (2.1), in order to break the second degeneracy. One then obtains a degenerate scalar-tensor theory with one scalar mode and two tensor modes. This is precisely how a theory "beyond Horndeski" was constructed in [4].

\section{DISFORMAL TRANSFORMATIONS IN OTHER CLASSES}

We now turn to the other classes of DHOST theories.

\section{A. Stability of all classes}

We have already pointed out the stability, under disformal transformations, of the sign (including zero) of $f$ and of $\alpha_{1}+\alpha_{2}$, which guarantes the stability of the classes I, II and III separately. We now consider the criteria that distinguish the subclasses within these classes. 
One can first notice the relation

$$
f-\alpha_{1} X=\mathcal{J}_{g}\left[\frac{1}{A+B X} \tilde{f}-\frac{X}{(A+B X)^{2}} \tilde{\alpha}_{1}\right]=\frac{\mathcal{J}_{g}}{A+B X}\left(\tilde{f}-\tilde{X} \tilde{\alpha}_{1}\right),
$$

where we have substituted the expression (4.22) for $h$ and the coefficient $\mathcal{T}_{11}$ in (4.28). If we start from a theory in Class Ib or in Class IIb, characterized by $\tilde{\alpha}_{1}=\tilde{f} / \tilde{X}$, the above relation implies that the disformally transformed theory verifies $\alpha_{1}=f / X$ and thus belongs to the same subclass, either Ib or IIb, as the original theory. Therefore, the classes Ia, Ib, IIa and IIb are separately stable.

We find the same properties for the subclasses in class III. Indeed, when $f=0$, we have $\alpha_{1}=\mathcal{J}_{g} \mathcal{T}_{11} \tilde{\alpha}_{1}$ and $\alpha_{2}=\mathcal{J}_{g} \mathcal{T}_{11} \tilde{\alpha}_{2}$. Therefore the signs of $\alpha_{1}$ and $\alpha_{1}+3 \alpha_{2}$ which distinguish the subclasses IIIa, IIIb and IIIc are conserved in a disformal transformation.

In summary, all the classes and subclasses that we have distinguished are separately stable under disformal transformations. In particular, the intersections of two classes or subclasses, when non empty, are also stable. This applies for instance to the intersection of Ia and IIIa, which contains $L_{4}^{\mathrm{bh}}$.

\section{B. Disformal transformations in Class IIa}

It is straightforward to specialize the general disformal transformations to class IIa. One just needs to impose that the tilted functions $\tilde{f}$ and the $\tilde{\alpha}_{I}$ satisfy the properties (3.25). Since Lagrangians in class IIa depend on three arbitrary functions, one can try to proceed as in class Ia by choosing a particular family that depends on a single arbitrary function and then produce generic theories by applying a disformal transformation. There is no natural candidate for this one-function family, in contrast with Horndeski. One could choose for example the family

$$
\tilde{f}=1, \quad \tilde{\alpha}_{1}=0, \quad \tilde{\alpha}_{3}=-\frac{4}{X^{2}}\left(1+2 X \alpha_{2}\right), \quad \tilde{\alpha}_{4}=\frac{2}{X^{2}}, \quad \tilde{\alpha}_{5}=\frac{8}{X^{3}}\left(1+2 X \alpha_{2}\right),
$$

which depends only on the arbitrary function $\tilde{\alpha}_{2}$.

One finds that the disformal transformation of this family leads, in particular, to

$$
f=A^{1 / 2}(A+B X)^{1 / 2}
$$

and

$$
\alpha_{2}=-\frac{A^{1 / 2}\left(A\left(B-\tilde{\alpha}_{2}\right)+B^{2} X\right)}{(A+B X)^{3 / 2}}
$$

We can solve the first equation to determine $B$ in terms of $A$ and $f$ :

$$
B=\frac{f^{2}-A^{2}}{A X}
$$

Substituting in (6.4) and solving for $\tilde{\alpha}_{2}$, we get

$$
\tilde{\alpha}_{2}=\frac{f^{2}}{A^{3} X}\left(f^{2}-A^{2}+X f \alpha_{2}\right) .
$$

One can then substitute these relations into the other coefficients obtained by disformal transformation. The coefficient $\alpha_{4}$, for instance, is particularly simple:

$$
\alpha_{4}=\frac{2}{f X^{2}}\left(A^{2}+4 X^{2} f_{X}^{2}-2 X f f_{X}\right) .
$$

In this way, one can determine the function $A$ in terms of $\alpha_{4}$ and $f$, and then $B$. Note however that this procedure works only if the above equation can be solved for $A^{2}$. This means that there will be restrictions on the theories generated by the family (6.2). 


\section{Disformal transformation in class IIIa}

Although class IIIa contains $L_{4}^{\mathrm{bH}}$, which depends on a single function, this Lagrangian cannot generate all theories in IIIa because it belongs to the intersection of IIIa and Ia and any of its transformed Lagrangians will also belong to this intersection. This means in particular that $L_{4}^{\mathrm{bH}}$ cannot be connected to Horndeski, as already pointed out in [9].

In class IIIa, $h=0$ and therefore the ratio between $\alpha_{1}$ and $\alpha_{2}$ remains conserved in a disformal transformation. This implies that one cannot choose a "seed" family with $\tilde{\alpha}_{1}=0$ or $\tilde{\alpha}_{2}=0$ in order to obtain generic theories of IIIa. Instead, one can try to take, for instance, the family

$$
\tilde{f}=0, \quad \tilde{\alpha}_{1}=1, \quad \tilde{\alpha}_{3}=0, \quad \tilde{\alpha}_{4}=-\frac{2}{X}, \quad \tilde{\alpha}_{5}=\frac{1+2 \tilde{\alpha}_{2}}{X^{2}\left(1+3 \tilde{\alpha}_{2}\right)},
$$

which depends on the arbitrary function $\tilde{\alpha}_{2}$. Applying a disformal transformation on this family, one gets

$$
\alpha_{1}=A^{3 / 2}(A+B X)^{-3 / 2}, \quad \alpha_{2}=A^{3 / 2}(A+B X)^{-3 / 2} \tilde{\alpha}_{2},
$$

which can be solved to give

$$
B=A \frac{1-\alpha_{1}^{2 / 3}}{X \alpha_{1}^{2 / 3}}, \quad \tilde{\alpha}_{2}=A^{-3 / 2}(A+B X)^{3 / 2} \alpha_{2} .
$$

Substituting into the expression for $\alpha_{3}$, one finds

$$
\frac{A_{X}}{A}=\frac{2 \alpha_{1} \alpha_{3}-4 \alpha_{3} \alpha_{1 X}}{6 \alpha_{1}\left(\alpha_{1}+2 \alpha_{2}\right)} .
$$

This enables us to determine $A$ and then $B$.

\section{LINK WITH KHRONOMETRIC AND MIMETIC THEORIES}

In this section we show that, aside Horndeski and its extension, quadratic DHOST theories also contain, as particular cases, other theories that have already been studied elsewhere. In the first part, we identify some "khronometric" theories in both class I and class II. In the second part, we discuss "mimetic" theories, associated with a non invertible disformal transformation.

\section{A. Khronometric theories}

Khronometric theories [20] represent a subset of Einstein-aether theories 21] for which the unit time-like vector $u^{\mu}$, which defines a special frame, is expressed as the normalized gradient of a scalar field,

$$
u_{\mu}=\frac{\nabla_{\mu} \phi}{\sqrt{-X}}
$$

Their dynamics is described by the action

$$
S=\int d^{4} x \sqrt{-g}\left(f R+K_{\rho \sigma}^{\mu \nu} \nabla_{\mu} u^{\rho} \nabla_{\nu} u^{\sigma}\right),
$$


with

$$
K_{\rho \sigma}^{\mu \nu} \equiv c_{1} g^{\mu \nu} g_{\rho \sigma}+c_{2} \delta_{\rho}^{\mu} \delta_{\sigma}^{\nu}+c_{3} \delta_{\sigma}^{\mu} \delta_{\rho}^{\nu}+c_{4} u^{\mu} u^{\nu} g_{\rho \sigma},
$$

where the $c_{a}$ are constant. This is the most general Lagrangian which depends only quadratically on the vector field $u^{\mu}$ and it is clearly invariant under arbitrary scalar field redefinitions $\phi \mapsto \psi(\phi)$. Since $f$ is constant, we can set $f=1$ without loss of generality.

Substituting (7.1) into (7.2)-(7.3), we find that the action (7.2) is of the form (2.1), with the functions

$$
\begin{aligned}
f & =1, \quad \alpha_{1}=-\frac{1}{X}\left(c_{1}+c_{3}\right), \quad \alpha_{2}=-\frac{1}{X} c_{2}, \quad \alpha_{3}=\frac{2}{X^{2}} c_{2}, \\
\alpha_{4} & =\frac{1}{X^{2}}\left(c_{1}+2 c_{3}+c_{4}\right), \quad \alpha_{5}=-\frac{1}{X^{3}}\left(c_{2}+c_{3}+c_{4}\right) .
\end{aligned}
$$

One immediately sees that the parameter $c_{1}$ can be absorbed in $c_{3}$ and $c_{4}$ by redefining $c_{3}+c_{1} \rightarrow c_{3}$ and $c_{4}-c_{1} \rightarrow c_{4}$. In the following, we will thus assume $c_{1}=0$ without loss of generality (one can always return to the original form by using the inverse redefinitions).

Among khronometric theories, which represent a subset of higher order theories, one can look for degenerate theories by examining the degeneracy conditions (3.13)-(3.15). Substituting (7.4), with $c_{1}=0$, into the first degeneracy condition yields

$$
D_{0}=\frac{4}{X}\left(c_{2}+c_{3}\right)\left(c_{4}-2\right)=0
$$

whose solutions yield several families of degenerate khronons.

\section{Khronons in Class I}

Let us first consider the case

$$
c_{2}+c_{3}=0
$$

which corresponds to class I, since this implies $\alpha_{1}+\alpha_{2}=0$. Substituting this condition into $D_{1}$ and $D_{2}$, one finds

$$
D_{1}=X D_{2}=-\frac{8}{X^{2}}\left(c_{2}-1\right)^{2} c 4
$$

which allows two possibilities.

The first family, characterized by

$$
\alpha_{1}=-\alpha_{2}=\frac{1}{X}, \quad \alpha_{3}=\frac{2}{X^{2}}, \quad \alpha_{4}=\frac{1}{X^{2}}\left(c_{4}-2\right), \quad \alpha_{5}=-\frac{c_{4}}{X^{3}},
$$

with $c_{4}$ arbitrary, belongs to the class Ib.

The second family, described by

$$
\alpha_{1}=-\alpha_{2}=\frac{c_{2}}{X}, \quad \alpha_{3}=\frac{2 c_{2}}{X^{2}}, \quad \alpha_{4}=-2 \frac{c_{2}}{X^{2}}, \quad \alpha_{5}=0
$$

with $c_{2}$ arbitrary, belongs to class Ia (except the case $c_{2}=1$ which is also in the previous family). 


\section{Khronometric Class II}

The second possibility to satisfy $D_{0}=0$ is given by

$$
c_{4}=2,
$$

which leads to

$$
D_{1}=X D_{2}=\frac{8}{X^{2}}\left(1+c_{3}\right)\left(3 c_{2}+c_{3}-2\right) .
$$

Once again, we get two families, but now belonging to class II.

The first family, corresponding to $c_{3}=-1$, is described by

$$
\alpha_{1}=\frac{1}{X}, \quad \alpha_{2}=-\frac{c_{2}}{X}, \quad \alpha_{3}=\frac{2 c_{2}}{X^{2}}, \quad \alpha_{4}=0, \quad \alpha_{5}=-\frac{c_{2}+1}{X^{3}},
$$

and depends on the arbitrary parameter $c_{2}$. These theories are in IIb, except for the case $c_{2}=1$, which is in IIa.

The second family, corresponding to $c_{3}=2-3 c_{2}$, also depends on the single parameter $c_{2}$ :

$$
\alpha_{1}=\frac{3 c_{2}-2}{X}, \quad \alpha_{2}=-\frac{c_{2}}{X}, \quad \alpha_{3}=\frac{2 c_{2}}{X^{2}}, \quad \alpha_{4}=\frac{6\left(1-c_{2}\right)}{X^{2}}, \quad \alpha_{5}=\frac{2\left(c_{2}-2\right)}{X^{3}} .
$$

These Lagrangians belong to IIb, except if $c_{2}=1$.

\section{Disformal transformations}

The set of khronometric theories is stable under the action of disformal transformations of the form

$$
\tilde{g}_{\mu \nu}=a g_{\mu \nu}+b u_{\mu} u_{\nu}
$$

i.e. for $A=a$ and $B=-b / X$, where $a$ and $b$ are constant.

Without loss of generality, one can restrict our analysis to one-parameter transformations such that $b=a-a^{-1}$, which preserve $f=1$ in the khronometric action (7.2). It is straightforward to study the action of disformal transformations of this type on the four degenerate khronometric families identified above. One finds that each theory of the first family (7.8) remains invariant. Each of the three other families is stable, the transformed theory being obtained by the following modification of the parameter $c_{2}$ :

$$
c_{2} \rightarrow\left(c_{2}-1\right) a^{2}+1 .
$$

In particular, one notes that, in the family (7.9), any theory with $c_{2}>0$ can be transformed into general relativity by choosing $a^{2}=1 /\left(1-c_{2}\right)$.

\section{B. Mimetic theories}

In previous sections, we have assumed that the disformal transformation (4.1) is invertible, in the sense that one can also express the metric $g_{\mu \nu}$ in terms of $\tilde{g}_{\mu \nu}$. When we relax this condition, one obtains the so-called "mimetic" theories, in analogy with the first model of this kind, investigated in [22], defined from a non-invertible disformal transformation of the Einstein-Hilbert action. 


\section{Non-invertible disformal transformation and symmetries}

By differentiating the expression

$$
\tilde{g}_{\mu \nu}=A(\phi, X) g_{\mu \nu}+B(\phi, X) \phi_{\mu} \phi_{\nu}
$$

one obtains

$$
\delta \tilde{g}_{\mu \nu}=F_{\mu \nu} \delta \phi+H_{\mu \nu}^{\alpha} \nabla_{\alpha} \delta \phi+J_{\mu \nu}^{\alpha \beta} \delta g_{\alpha \beta}
$$

with

$$
\begin{aligned}
F_{\mu \nu} & =A_{\phi} g_{\mu \nu}+B_{\phi} \phi_{\mu} \phi_{\nu} \\
H_{\mu \nu}^{\alpha} & =2\left(A_{X} g_{\mu \nu}+B_{X} \phi_{\mu} \phi_{\nu}\right) \phi^{\alpha}+B\left(\phi_{\nu} \delta_{\mu}^{\alpha}+\phi_{\mu} g_{\nu}^{\alpha}\right) \\
J_{\mu \nu}^{\alpha \beta} & =A \delta_{(\mu}^{\alpha} \delta_{\nu)}^{\beta}-\phi^{\alpha} \phi^{\beta}\left(A_{X} g_{\mu \nu}+B_{X} \phi_{\mu} \phi_{\nu}\right) .
\end{aligned}
$$

As discussed in [4], the disformal transformation is non invertible, i.e. $g_{\mu \nu}$ cannot be determined from $\tilde{g}_{\mu \nu}$, if the determinant of the Jacobian matrix $J_{\mu \nu}^{\alpha \beta} \equiv \frac{\partial \tilde{g}_{\mu \nu}}{\partial g_{\alpha \beta}}$ vanishes. This happens when $J_{\mu \nu}^{\alpha \beta}$ admits a null vector $v_{\alpha \beta}$ such that

$$
J_{\mu \nu}^{\alpha \beta} v_{\alpha \beta}=0 .
$$

It is straightforward to check that the combination

$$
v_{\alpha \beta}=A_{X} g_{\alpha \beta}+B_{X} \phi_{\alpha} \phi_{\beta}
$$

is a null vector of the Jacobian matrix, provided the functions $A$ and $B$ verify

$$
B_{X}=\frac{A-X A_{X}}{X^{2}}
$$

After integration, this yields

$$
B=-\frac{A}{X}+\mu(\phi)
$$

corresponding to the disformal transformation

$$
\tilde{g}_{\mu \nu}=A(\phi, X)\left(g_{\mu \nu}-\frac{1}{X} \phi_{\mu} \phi_{\nu}\right)+\mu(\phi) \phi_{\mu} \phi_{\nu}
$$

Note that if we insert (7.24) into (4.5), one gets $\tilde{X}=1 / \mu(\phi)$, which shows that $\tilde{X}$ does not depend on $X$.

\section{Mimetic action}

If we start from an action of the form

$$
\tilde{S}\left[\phi, \tilde{g}_{\mu \nu}\right]=\int d^{4} x \sqrt{-\tilde{g}}\left(\tilde{f}(\phi) \tilde{R}+\alpha_{I}(\phi) \tilde{L}_{I}^{\phi}\right),
$$

and substitute (7.25), we obtain a new action $S$, given as a functional of $g_{\mu \nu}$ and $\phi$. This leads to a subclass of our DHOST theories with particular properties. This procedure has been used 
in 22] for the Einstein-Hilbert action, i.e. $\tilde{f}=1$ and $\tilde{\alpha}_{I}=0$, with the disformatl transformation characterized by $A=X$ and $B=0$, to introduce the model of mimetic dark matter. It has been extended in [23] to a general non-invertible transformation, with (7.24). In contrast with the generic case where the disformal transformation is invertible, the number of degrees of freedom is not necessarily the same for $\tilde{S}$ and $S$. In particular, if $\tilde{S}$ is the Einstein-Hilbert action, with only two degrees of freedom, one ends up with three degrees of freedom for $S$, as discussed in [22] and [23].

Interestingly, the mimetic action $S$ is invariant under the local symmetry

$$
\delta \phi=0 \quad \text { and } \quad \delta g_{\mu \nu}=\varepsilon v_{\mu \nu}=\varepsilon\left(A_{X} g_{\mu \nu}+B_{X} \phi_{\mu} \phi_{\nu}\right),
$$

where $\varepsilon$ is an infinitesimal space-time function. This symmetry follows immediately from (7.17), together with the property that $v_{\mu \nu}$ is a null eigenvector of the Jacobian matrix.

In the Hamiltonian framework, such a symmetry implies the existence of an extra first class constraint in addition to the usual Hamiltonian and momentum constraints associated with diffeomorphism invariance. This is in contrast with the standard quadratic DHOST theories, for which the extra constraints are generically second class, as shown in [8]. One of these second class constraints is necessary to eliminate the Ostrogradski ghost and we thus expect that, even though mimetic theories contain three degrees of freedom, the Ostrogradski ghost is still present.

This is indeed the case for the simplest model of mimetic gravity, obtained from Einstein-Hilbert with $A=X$ and $B=0$. In that case, the symmetry (7.27) reduces to an invariance under conformal transformations of $g_{\mu \nu}$. In the Hamiltonian description, this symmetry is necessarily associated to a first class constraint. Following the analysis of [8], and introducing the conjugate momenta $\pi^{i j}$ and $p_{*}$ of $h_{i j}$ and $A_{*}$, respectively, one can show that the primary constraint reduces to

$$
\Psi \equiv \gamma_{i j} \pi^{i j}-\frac{1}{2} p_{*},
$$

which is indeed the generator of infinitesimal conformal transformations. As a consequence, the primary constraint is first class and it Poisson commutes with the Hamiltonian and momentum constraints. Hence, there is no secondary constraint that eliminates the Ostrogradski ghost. This has already been noticed in [24] and we expect this to remain true for any mimetic-like theory.

\section{CONCLUSIONS}

The degeneracy, in the generalized sense introduced in [7], of scalar-tensor Lagrangians is a powerful tool to classify viable alternative theories of gravity. In the present work we have revisited all the quadratic DHOST theories identified in [7] and studied how they transform under generalized disformal transformations. In order to do so, we have obtained the general transformation laws of the six arbitrary functions that appear in the Lagrangian of these theories. This shows that any quadratic DHOST theory is transformed, via disformal transformation, into another quadratic DHOST theory, up to terms that are at most linear in $\phi_{\mu \nu}$ (which do not affect the degeneracy of the theory). Moreover, we have found that the three main classes of quadratic DHOST theories, as well as the two or three subclasses within each, are all stable under disformal transformations. One of these subclasses (class Ia) contains the theories "generated" from the (quadratic) Horndeski Lagrangian via disformal transformations, .

Two disformally related theories describe distinct physics if matter is assumed to be minimally coupled for both theories. Conversely, a given scalar tensor theory can be described by different disformally related Lagrangians, provided the coupling to matter is modified accordingly. In this sense, the situation is very similar to traditional scalar-tensor theories for which one can use the 
Jordan frame, in which matter is minimally coupled to the metric, or the Einstein frame, where the gravitational dynamics is described by the usual Einstein-Hilbert Lagrangian but at the price of a non-minimal coupling of matter to the metric. Similarly, for the quadratic DHOST theories in class Ia, one can work either in the Jordan frame, where matter is minimally coupled but the equations of motion are in general higher order, or in the "Horndeski" frame where the equations of motion are second order but with a non-minimal coupling of matter to the metric.

Apart Horndeski and its extension, we have also recognized other known theories among the quadratic DHOST theories. Khronometric theories, which are a sub-class of Einstein-aether theories where the unit vector is proportional to the gradient of a scalar field, lead to higher order scalar-tensor theories when covariantized. These covariantized theories are in general not degenerate but we have found that a subset of them are indeed degenerate. Our theories also encompass mimetic gravity, which is obtained from the Einstein-Hilbert Lagrangian via a specific, non invertible, disformal transformation.

Quadratic DHOST theories also contain theories that cannot be related, up to disformal disformations, to known theories, at least to our knowledge, and thus seem to represent genuinely new scalar-tensor theories, independently of their specific coupling to matter. It would be interesting to study the cosmology of these new theories by using, for instance, the general formalism developed in [25, 26].

Note added: the paper [12], which appeared on arXiv during the preparation of this manuscript, also studies the theories introduced in [7] and has some partial overlap with the present work, in particular concerning the disformal transformations in class Ia.

[1] M. Ostrogradsky. Mem. Ac. St. Petersbourg VI 4 (1850) 385; R. P. Woodard, Lect. Notes Phys. 720, 403 (2007) [astro-ph/0601672]; R. P. Woodard, arXiv:1506.02210 [hep-th].

[2] G. W. Horndeski, "Second-order scalar-tensor field equations in a four-dimensional space," Int. J. Theor. Phys. 10, 363 (1974).

[3] C. Deffayet, X. Gao, D. A. Steer and G. Zahariade, "From k-essence to generalised Galileons," Phys. Rev. D 84, 064039 (2011) [arXiv:1103.3260 [hep-th]].

[4] M. Zumalacarregui and J. Garcia-Bellido, "Transforming gravity: from derivative couplings to matter to second-order scalar-tensor theories beyond the Horndeski Lagrangian," Phys. Rev. D 89, 064046 (2014) [arXiv:1308.4685 [gr-qc]].

[5] J. Gleyzes, D. Langlois, F. Piazza and F. Vernizzi, "Healthy theories beyond Horndeski," Phys. Rev. Lett. 114, no. 21, 211101 (2015) [arXiv:1404.6495 [hep-th]].

[6] J. Gleyzes, D. Langlois, F. Piazza and F. Vernizzi, "Exploring gravitational theories beyond Horndeski," JCAP 1502, 018 (2015) [arXiv:1408.1952 [astro-ph.CO]].

[7] D. Langlois and K. Noui, "Degenerate higher derivative theories beyond Horndeski: evading the Ostrogradski instability," JCAP 1602, no. 02, 034 (2016) [arXiv:1510.06930 [gr-qc]].

[8] D. Langlois and K. Noui, "Hamiltonian analysis of higher derivative scalar-tensor theories," arXiv:1512.06820 [gr-qc].

[9] M. Crisostomi, M. Hull, K. Koyama and G. Tasinato, "Horndeski: beyond, or not beyond?," arXiv:1601.04658 [hep-th].

[10] J. D. Bekenstein, "The Relation between physical and gravitational geometry," Phys. Rev. D 48, 3641 (1993) [gr-qc/9211017].

[11] D. Bettoni and S. Liberati, "Disformal invariance of second order scalar-tensor theories: Framing the Horndeski action," Phys. Rev. D 88, 084020 (2013) [arXiv:1306.6724 [gr-qc]].

[12] M. Crisostomi, K. Koyama and G. Tasinato, "Extended Scalar-Tensor Theories of Gravity," arXiv:1602.03119 [hep-th]. 
[13] H. Motohashi and J. White, "Disformal invariance of curvature perturbation," arXiv:1504.00846 [gr-qc].

[14] G. Domenech, A. Naruko and M. Sasaki, "Cosmological disformal invariance," JCAP 1510, no. 10, 067 (2015) doi:10.1088/1475-7516/2015/10/067 [arXiv:1505.00174 [gr-qc]].

[15] S. Tsujikawa, "Cosmological disformal transformations to the Einstein frame and gravitational couplings with matter perturbations," Phys. Rev. D 92, no. 6, 064047 (2015) doi:10.1103/PhysRevD.92.064047 [arXiv:1506.08561 [gr-qc]].

[16] G. Domenech, S. Mukohyama, R. Namba, A. Naruko, R. Saitou and Y. Watanabe, "Derivativedependent metric transformation and physical degrees of freedom," Phys. Rev. D 92, no. 8, 084027 (2015) [arXiv:1507.05390 [hep-th]].

[17] W. T. Emond and P. M. Saffin, "Disformally self-tuning gravity," arXiv:1511.02055 [hep-th].

[18] T. Fujita, X. Gao and J. Yokoyama, "Spatially covariant theories of gravity: disformal transformation, cosmological perturbations and the Einstein frame," JCAP 1602, no. 02, 014 (2016) doi:10.1088/14757516/2016/02/014 [arXiv:1511.04324 [gr-qc]].

[19] X. Gao, "Unifying framework for scalar-tensor theories of gravity," Phys. Rev. D 90, 081501 (2014) doi:10.1103/PhysRevD.90.081501 [arXiv:1406.0822 [gr-qc]].

[20] D. Blas, O. Pujolas and S. Sibiryakov, "Models of non-relativistic quantum gravity: The Good, the bad and the healthy," JHEP 1104, 018 (2011) [arXiv:1007.3503 [hep-th]].

[21] T. Jacobson and D. Mattingly, "Gravity with a dynamical preferred frame," Phys. Rev. D 64, 024028 (2001) [gr-qc/0007031].

[22] A. H. Chamseddine and V. Mukhanov, "Mimetic Dark Matter," JHEP 1311, 135 (2013) [arXiv:1308.5410 [astro-ph.CO]].

[23] N. Deruelle and J. Rua, "Disformal Transformations, Veiled General Relativity and Mimetic Gravity," JCAP 1409, 002 (2014) [arXiv:1407.0825 [gr-qc]].

[24] M. Chaichian, J. Kluson, M. Oksanen and A. Tureanu, "Mimetic dark matter, ghost instability and a mimetic tensor-vector-scalar gravity," JHEP 1412, 102 (2014) [arXiv:1404.4008 [hep-th]].

[25] J. Gleyzes, D. Langlois, F. Piazza and F. Vernizzi, "Essential Building Blocks of Dark Energy," JCAP 1308, 025 (2013) [arXiv:1304.4840 [hep-th]].

[26] J. Gleyzes, D. Langlois and F. Vernizzi, "A unifying description of dark energy," Int. J. Mod. Phys. D 23, no. 13, 1443010 (2015) [arXiv:1411.3712 [hep-th]]. 